\title{
1 alpha, 25-dihydroxylvitamin D3 promotes Bacillus Calmette- Guérin immunotherapy of bladder cancer
}

\author{
Jong-Wei Hsu ${ }^{1,2}$, Peng-Nien Yin ${ }^{1}$, Ronald Wood ${ }^{3}$, James Messing ${ }^{4}$, Edward Messing ${ }^{1}$, \\ and Yi-Fen Lee L,2 $^{1,2}$ \\ ${ }^{1}$ Department of Urology, University of Rochester, Rochester, New York, USA \\ 2 Department of Pathology, University of Rochester, Rochester, New York, USA \\ ${ }^{3}$ Department of Obstetrics and Gynecology, University of Rochester, Rochester, New York, USA \\ ${ }^{4}$ Cornell University, Ithaca, New York USA \\ Correspondence to: Yi-Fen Lee, email: yifen_lee@urmc.rochester.edu \\ Keywords: vitamin D, Bacillus Calmette-Guérin, bladder cancer, immunotherapy, interleukin 8 \\ Received: October 14, $2013 \quad$ Accepted: November 17, $2013 \quad$ Published: November 19, 2013
}

This is an open-access article distributed under the terms of the Creative Commons Attribution License, which permits unrestricted use, distribution, and reproduction in any medium, provided the original author and source are credited.

\section{ABSTRACT:}

Bacillus Calmette-Guérin (BCG), a vaccine against tuberculosis(TB), has been used and proven to be one of the most effective treatments for non-muscle invasive bladder cancer (BCa). However, the mechanisms of BCG action have not been completely understood, thereby limiting the improvement of BCG therapy. Vitamin $D$ deficiency has been associated with a high risk of TB infection, and the beneficial effect of UV exposure in TB patients was proven to be mediated via activation of vitamin D signals of innate immune cells. Thus, vitamin D signals might be involved in mediating BCG immunotherapy. To test this hypothesis, we examined the impact of 1alpha, 25-dihydroxyvitamin D3 (1,25-VD) on BCG-induced response in BCa cells and macrophage cells. Our data revealed that 1,25-VD promotes BCG-induced interleukin 8 (IL-8) secretion by BCa cells, consequently inducing the migration of macrophage, THP-1. This THP-1 cell migration promoted by 1,25-VD can be blocked by IL-8 neutralized antibody. Furthermore, 1,25-VD increased BCG-induced expression of macrophage markers in THP-1 cell, and enhanced the BCG-induced THP-1 cytotoxicity against low-grade BCa cells. Importantly, a pre-clinical trial using the $\mathrm{N}$-butyl- $\mathrm{N}$-(4hydroxybutyl)-nitrosamine (BBN)-induced BCa mouse model revealed that intravesical co-treatment of 1,25-VD with BCG can prolong mice survival. These data demonstrate a novel mechanism by which 1,25-VD promotes BCG-mediated anti-BCa pathways and provides a platform for improving BCG efficacy with combination of 1,25-VD.

\section{INTRODUCTION}

Bladder cancer $(\mathrm{BCa})$ is the fourth leading cancer in prevalence and the eleventh leading cancer cause of death in American men [1]. BCa ranks highest in cost from diagnosis to death per patient [2]. About $80 \%$ of $\mathrm{BCa}$ patients are diagnosed with non-muscle invasive tumors [3], which include carcinoma in situ (CIS); Ta stage (lowgrade non-invasive); and $\mathrm{T} 1$ stage (invasiveness into lamina propria). For non-muscle invasive $\mathrm{BCa}$, patients are usually treated with a transurethral resection of bladder tumor (TURBT) to remove the existing tumors. Then, patients undergo a series of intravesical therapies, such as Bacillus Calmette-Guérin (BCG) or mitomycin $\mathrm{C}$, to eliminate the residual cancer cells and prevent recurrence. BCG was initially developed as a vaccine for tuberculosis (TB) in 1921 and was used as an adjuvant immunotherapy for BCa in 1976 [4]. Since then, BCG has been the most effective adjuvant treatment for non-muscle invasive $\mathrm{BCa}$. It was proven that $\mathrm{BCG}$ can prevent tumor recurrence and slow the progression to muscle invasive stage [5], thereby yielding a higher survival rate than TURBT surgery alone [6]. The mechanism of BCG in $\mathrm{BCa}$ therapy is known to involve both innate and adaptive immune cells. In brief, BCG is given intravesically, and the BCG adheres to urothelial cells through fibronectin 
[7] and alpha 5 beta 1 integrin receptors [8] followed by internalization. Urothelial cells are then induced to secrete cytokines, including IL-6, IL-8, and TNF-alpha, that recruit neutrophils and monocytes/macrophages [9]. Neutrophils play multiple roles in BCG therapy, through directly eliminating tumor cells by secreting TNF-related apoptosis-inducing ligand (TRAIL) [10] and indirectly contributing to tumor elimination by secreting cytokines to recruit effector cells, such as T cells and NK cells [5].

In addition to neutrophils, several lines of evidence suggest that macrophage actively mediates BCG-induced anti-BCa activity. Following BCG installation, increased numbers of macrophage, along with $\mathrm{T}$ cells, and natural killer (NK) cells are observed in $\mathrm{BCa}$ infiltrates. In addition to antigen presenting cells, macrophage also acts as a cytotoxic effector against $\mathrm{BCa}$ cells by releasing INF-alpha, INF-gamma, and NO after BCG stimulation [11]. Those effector molecules are known to induce cell apoptosis [11].

IL-8 is a potent chemoattractant for proinflammatory mediator, and it is expressed by immune cells and epithelial cells in response to BCG [12]. Urinary IL-8 can predict BCG responsiveness [13, 14]. Also, IL-2 secreted from BCG-stimulated macrophages contributes to the maturation of natural killer (NK) cells and cytotoxic $\mathrm{T}$ cells that serve as effector cells to kill bladder tumor cells [15]. BCG stimulates innate immune cells to secrete a panel of cytokine to further recruit adaptive immune cells, such as CD4+ and CD8+ T cells, as well as NK cells [16]. Then, Th1 cytokines are produced by both innate and adaptive immune cells, including IL-2, TNF-alpha, and INF-gamma, and these cytokines are required for eradicating tumor cells [5].

One recent study confirmed that the BCG-induced immune response in both innate and adaptive immune cells is critical for BCG efficacy where pre-existing BCG immunity improves BCG immune response to tumors in mice. The results were further proven in $\mathrm{BCa}$ patients showing that patients with pre-existing BCG response, determined by positive purified protein derivative (PPD) skin test, had a significantly better recurrence-free survival after standard BCG therapy than patients with a negative PPD skin test $[17,18]$.

The clinical $\mathrm{BCG}$ response rate in $\mathrm{BC}$ patients is $50-70 \%$, and a significant amount of patients fail BCG therapy. Currently, there is no biomarker to predict the patient's BCG responsiveness. Also, the majority of BCG patients developed mild cystitis, including urgency, malaise, and fever [19]. Interestingly, many reports suggest BCG-induced symptoms are associated with therapeutic efficacy [5], including cytokine release in the urine [20] and cystitis symptoms. Occasionally, patients may suffer from severe side effects such as a lifethreatening sepsis. Once severe symptoms occur, BCG therapy may be postponed or withheld, and patients need to take long-term anti-tuberculosis treatment to reduce the potential side effects [21]. Better understanding of BCG functional mechanism is indeed greatly needed for improving BCG therapy efficacy as well as development of biomarkers for a better selection of $\mathrm{BCa}$ patients before and during the therapy.

Accumulating evidence suggests that vitamin $\mathrm{D}$ is beneficial to $\mathrm{BCG}$ vaccination against $\mathrm{TB}$. Low serum vitamin $\mathrm{D}$ level is associated with susceptibility to TB infection [22]. Recent studies found that activation of toll-like receptor $1 / 2$ (TLR1/2) by mycobacteria, like $\mathrm{BCG}$, activates vitamin $\mathrm{D}$ signals by up-regulation of the expression of vitamin D receptor (VDR) and CYP27 (the enzyme that converts 25-dihydroxyvitamin $\mathrm{D}$ to active 1,25 -VD), thus leading to the induction of antimicrobial function [23]. VDR expression is detected in human superficial transitional cell carcinoma (TCC) of bladder [24]. Recent studies also indicated that low vitamin D level is associated with the risk of $\mathrm{BCa}$ [25], and high vitamin D plasma level protects against $\mathrm{BCa}$. Therefore, it is likely that vitamin D signaling may contribute to $\mathrm{BCa}$ disease progression and response to BCG immunotherapy.

We examined the effects of $1,25-\mathrm{VD}$ on BCGstimulated cytokine production, macrophage infiltration, and the cytotoxicity against $\mathrm{BCa}$ exerted by infiltrated macrophage. Importantly, we performed a pre-clinical trial to test if $1,25-\mathrm{VD}$ can promote BCG efficacy in a carcinogen-induced $\mathrm{BCa}$ mouse model.

\section{RESULTS}

\section{1,25-VD promotes IL-8 secretion by BCa cells}

To understand if vitamin D signaling occurs in the human bladder, the expression and distribution of vitamin D receptor (VDR)-- the key factor that modulates vitamin D activity-- were examined in human bladder samples by immunohistochemistry (IHC) staining. As shown in Fig. 1A, we successfully detected VDR expression in both normal human urothelium and carcinoma in situ (CIS) so as to suggest a potential vitamin $\mathrm{D}$ action in the human bladder. Moreover, we also detected VDR expression levels in various bladder cell lines, including immortalized normal urothelial cell line; SV-HUC; and two high grade BCa cell lines; T24 (grade III); TCC-SUP (grade IV) and one low grade HT1197 by Western blot analysis. VDR is expressed in all the cells we examined with higher abundance in HT1197 and T24 cells and much less expressed in SV-HUC cells (Fig. 1B).

Following BCG instillation, urothelial cells secrete a specific profile of pro-inflammatory cytokines, including IL-6, IL-8, and TNF-alpha [28, 29], which induce the first wave of immune response, innate immune cell infiltration. To test if $1,25-\mathrm{VD}$ influences $\mathrm{BCa}$ cells cytokine production, we examined the levels of IL-6, IL-8, and 
TNF-alpha in BCa cells, TCC-SUP and T24 in response to BCG and/or 1,25-VD; SV-HUC immortalized cells were used as a control. As shown in Figure 2, BCG induced significant IL- 8 production, and 1,25-VD can promote further the BCG-induced IL-8 secretion in both BCa cell lines. In contrast, the immortalized SV-HUC cells have very limited cytokine secretion in response to BCG and/ or 1,25-VD (Fig. 2). Contrary to IL-8, we found 1,25VD has very little influence on the BCG-induced IL-6 and TNF-alpha secretion (supplement data, Figs. 1A and 1B) in most of the cell lines we tested.

\section{1,25-VD promotes BCG-induced macrophage migration via increasing II-8 secretion}

The pro-inflammatory cytokine secretion by $\mathrm{BCa}$ cells following BCG instillation would consequently induce innate immune cells infiltration into the bladder wall, and this first wave immune response is required for a successful BCG therapy [30]. Therefore, the 1,25VD induction of IL- 8 secretion by BCa cells could then promote BCG-induced innate immune cell migration. To test this, we performed a transwell migration assay whereby we treated TCC-SUP cells with vehicle control, 1,25-VD, BCG, and BCG+1,25-VD for two hours, then replaced with culture media for 22 hours incubation. Afterwards, the conditioned media that served as chemo-attractant sources were collected for migration of monocyte/macrophage THP-1 cells. We found that BCG treatment induced more THP-1 cell migration than vehicle control. Importantly, $\mathrm{BCG}+1,25-\mathrm{VD}$ treatment induced

A

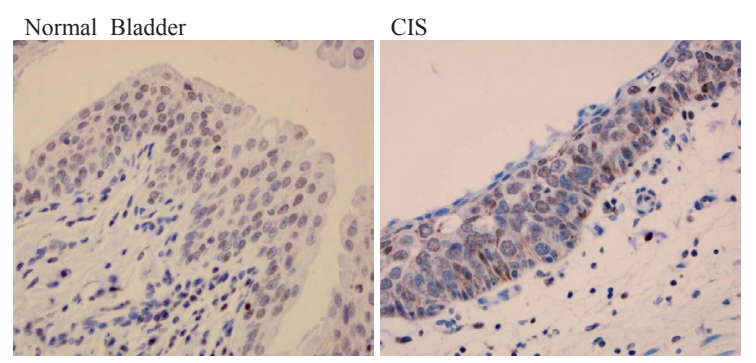

B

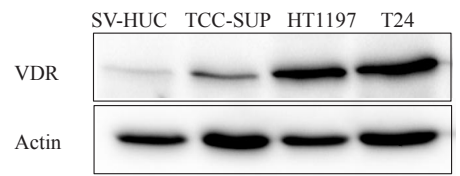

Figure 1: Detection of VDR expression in human bladder. (A) VDR protein expression was detected in human bladder tissues including benign bladder and bladder carcinoma in situ (CIS) by IHC staining. (B) VDR expression in bladder cell lines, including immortalized normal urothelial cell line SVHUC and three BCa cell lines, TCC-HUC (grade IV), HT1197 (low grade) and T24 (grade III) cells by Western blotting assay. $\beta$-actin was used as a loading control. statistically more THP-1 migration than BCG alone (Fig. 3 upper panel).

To confirm whether the THP-1 cell migration induced by $\mathrm{BCG}+12,5-\mathrm{VD}$ treatment was due to the elevated IL-8, IL-8 neutralizing antibody that blocks IL-8 action was employed. As expected, we found that IL-8 neutralizing antibody significantly decreased both BCGand $\mathrm{BCG}+1,25-\mathrm{VD}$-induced THP cell migration (Fig. 3, lower panel). It was noted that IL-8 neutralizing antibody can suppress both BCG and BCG+1,25-VD induced THP1 migration to similar levels, suggesting Il-8 is the key pro-inflammatory cytokine that is involved in the THP-1 migration to $\mathrm{BCa}$ cells.

\section{1,25-VD increased the expression of activated macrophage markers and inflammatory IL-8 expression}

Vitamin D signaling is known to be critical for controlling mycobacteria infection through the regulation of TLR signal in innate immune cells, such as macrophage. Given the critical roles of macrophage in initiating an effective response to BCG therapy, we determined the expression of activated macrophage markers and cytokine secretion in THP-1 cells in responses to BCG and/or 1,25-VD. Three activated macrophage markers, CD163, Chitotriosidase (CHIT) and CD68, were examined when THP-1 cells were treated with BCG and/or 1,25-VD. We found that all three macrophage markers were significantly induced by BCG treatment. CD163 and CHIT, but not CD68, expression levels were further enhanced by

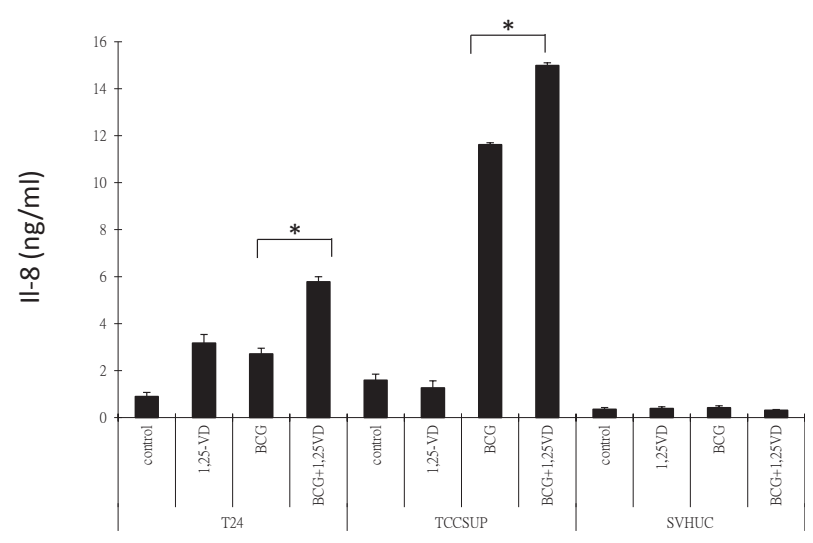

Figure 2: 1,25-VD promotes BCG-induced proinflammatory IL-8 cytokine secretion. $2 \times 10^{5}$ TCCSUP, T24, and SV-HUC cells were seeded in a 24-well culture plate in $10 \%$ FBS RPMI medium. Cells were then treated with vehicle (ethanol), $100 \mathrm{nM} \mathrm{1,25-VD,} 2 \times 10^{5} \mathrm{CFU}$ BCG, or in combination of 1,25-VD and BCG. Culture media were replaced with fresh media after 2 hours treatment of drugs, and cells were then cultured for another 22 hours. The supernatants were collected for assaying IL-8 pro-inflammatory cytokine secretion by ELISA (eBioscience). 
additional 1,25-VD treatment (Fig. 4) in THP-1 cells. With respect to the elevated macrophage marker expression, BCG also induces morphological alteration in THP-1 cells where the majority of BGC-treated THP-1 cells were attached with a spindle-like morphology as compared to undifferentiated state where cells are round and suspended as shown in vehicle treated cells. Importantly, we found more spindle-like cells were attached suggesting 1,25VD promotes BCG-induced macrophage differentiation; unexpectedly, we did not see too much alteration in the 1,25-VD treated THP-1 cells.

Among those markers, CD163 was particularly interesting; it was induced significantly in the $1,25-\mathrm{VD}+\mathrm{BCG}$ group. CD163 is a cell surface receptor for bacteria and functions as an innate immune sensor for bacteria. The recognition of bacteria by CD163 could enhance inflammatory cytokine production in THP-1 cells [31]. Therefore, we tested if the combination of $1,25-\mathrm{VD}$

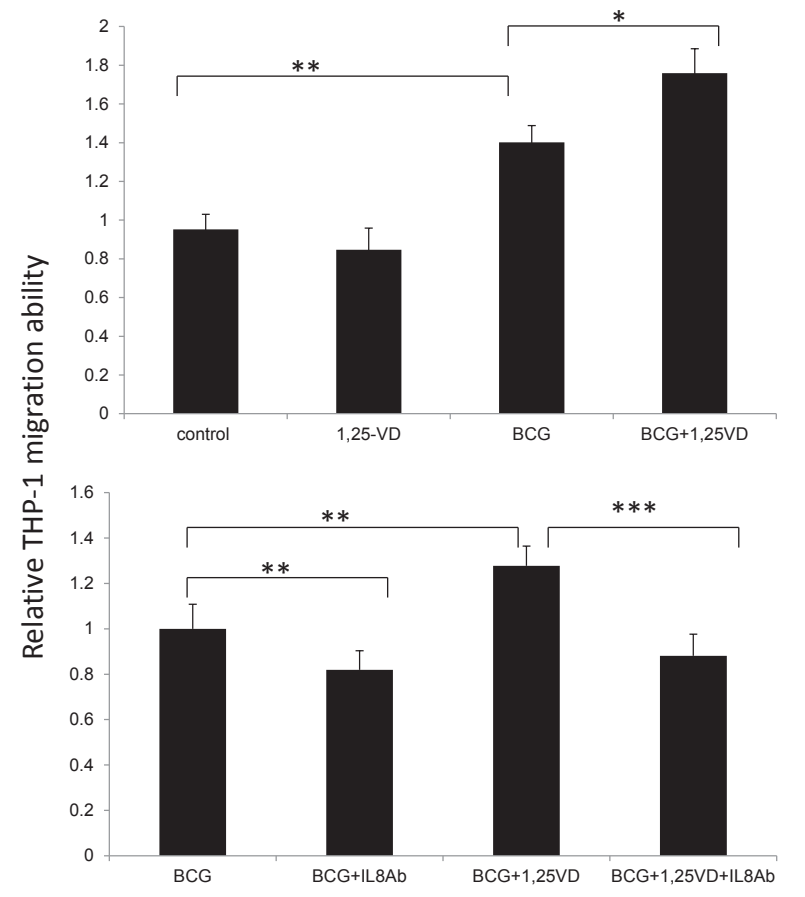

Figure 3: 1,25-VD promotes THP-1 cell migration through IL-8. (A) 1,25-VD promotes BCG induced THP-1 cell migration by a transwell migration assay. TCC-SUP cells were treated with vehicle (ethanol), $100 \mathrm{nM} 1,25-\mathrm{VD}, 2 \times 10^{5} \mathrm{CFU}$ $\mathrm{BCG}$, or in combination of $1,25-\mathrm{VD}$ and BCG. Culture media were replaced with fresh media after 2 hours' treatment of drugs, then cells were cultured for another 22 hours. The conditioned media were collected and loaded in the lower chamber, and Thp1 cells after serum-free starvation were added to transwell insert with $5 \mu \mathrm{m}$ pore. After 24 hours the infiltrating THP-1 cells in the lower chamber were collected and counted. (B) Blockage of IL-8 by neutralized antibody reverses 1,25-VD-induced THP-1 migration. A transwell migration assay was performed as described in A, except adding IL-8 neutralizing antibody (20 $\mu \mathrm{g} / \mathrm{ml}$ ) with BCG and BCG+1,25-VD treated TCC-SUP cells. For statistical analysis, differences in mean values between two groups were analyzed by two-tailed Student's test. Results are the mean \pm SEM of three experiments. with BCG could further induce cytokine secretion by THP-1 cells. Since we had shown in Figure 2 that 1,25VD enhanced BCG-induced IL-8 production in $\mathrm{BCa}$ cells, we now tested if $1,25-\mathrm{VD}$ can further promote IL-8 production in THP-1. As shown in Fig. 4B, BCG increased IL-8 expression at both mRNA and protein levels, and this induction can be further enhanced by $1,25-\mathrm{VD}$. These data suggested that 1,25-VD promotes macrophage maturation and activity in response to BCG.

\section{1,25-VD promotes BCG-induced THP-1 cytotoxicity against $\mathrm{BCa}$ cells}

It has been reported that $\mathrm{BCG}$ can induce macrophage cytotoxicity against $\mathrm{BCa}$ cells $[11,32]$. As we showed, 1,25-VD can promote BCG-induced macrophage activation and cytokine production. We therefore tested if $1,25-\mathrm{VD}$ can promote BCG induced macrophage cell cytotoxicity against BCa cells. We treated THP-1 cells with $\mathrm{BCG}$ and/or 1,25-VD, and conditioned media were collected for testing cytotoxicity against $\mathrm{BCa}$ cells. Two BCa cell lines were used: TCC-SUP and HT1197. As expected, BCG-treated THP-1 conditioned medium inhibited both TCC-SUP and HT1197 BCa cells growth significantly. Interestingly, 1,25-VD increased the BCGmediated macrophage cytotoxicity against low-grade HT1197 cells but it only slightly increased cytotoxicity against high-grade TCC-SUP cells (Fig. 5). This cell specific 1,25-VD impact on BCG-induced macrophage cytotoxicity against $\mathrm{BCa}$ might be due to the lack of an optimal cell environment for 1,25-VD action, and low abundance of VDR expression in TCC-SUP cells (Fig. 1) as compared to HT1197 cells might be associated with less 1,25-VD impact on BCG-induced macrophage cytotoxicity against $\mathrm{BCa}$ cells.

\section{BCG and 1,25-VD prolongs survival in BBN induced BCa mice}

Our in vitro results suggest that $1,25-\mathrm{VD}$ promotes BCG-induced innate immune responses and recruits more macrophage cells toward $\mathrm{BCa}$ cells, thereby suppressing $\mathrm{BCa}$ cell growth. To further examine whether the BCG and 1,25-VD combination treatment can improve BCG efficacy in vivo, a carcinogen (BBN)-induced $\mathrm{BCa}$ mouse model was used [33]. BBN is a DNA damaging carcinogen derived from tobacco smoke. It induces $\mathrm{BCa}$ arising from urothelium in mice and represents a slowly progressing $\mathrm{BCa}$ mouse model in which mice develop an early precancerous lesion that is similar to CIS (carcinoma in situ) in $\mathrm{BCa}$ patients. A total of 24 mice were given $\mathrm{BBN}$ in their drinking water to induce BCa. Following a 12-week $\mathrm{BBN}$ treatment, mice were randomly grouped into four treatments: vehicle control (100\% ethanol); 1,25-VD (100 $\mathrm{nM})$; BCG (1x10 $\left.10^{6} \mathrm{FU}\right)$; or BCG+1,25 for 6 weeks, while 
mice survival was monitored and recorded.

The results showed that the BCG-treated group had only a slightly better overall survival rate than the control or 1,25-VD treated groups. Importantly, the combination of 1,25-VD with BCG greatly improved overall mice survival. The dead mice were found to have enlarged kidneys with hydronephrosis and non-transparent bladder tumor mass indicating that death was attributed to the $\mathrm{BCa}$-induced obstruction of the urinary tracts. This pre- clinical data supports that combination of BCG with 1,25VD results in better treatment efficacy than single drug treatment. Further determination of optimal dosages for BCG and 1,25-VD is highly desirable (Fig. 6).

\section{DISCUSSION}

Combination therapy in which multiple drugs targeting the same cellular pathways could function

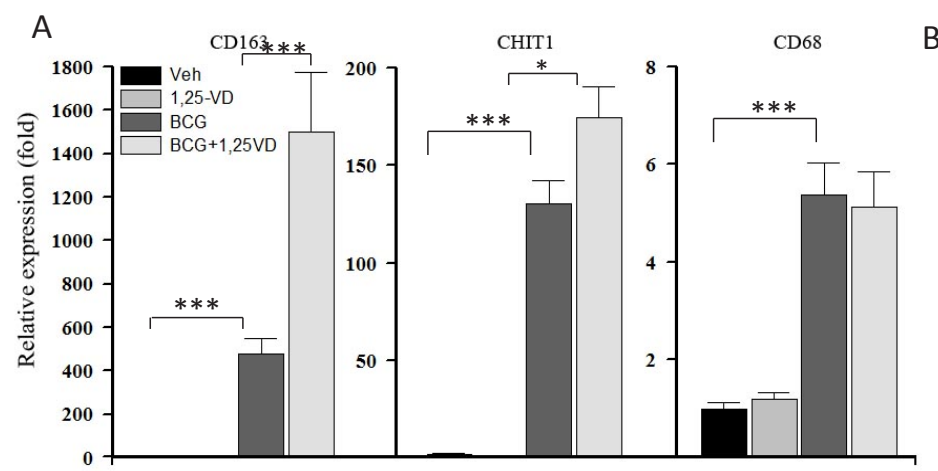

B
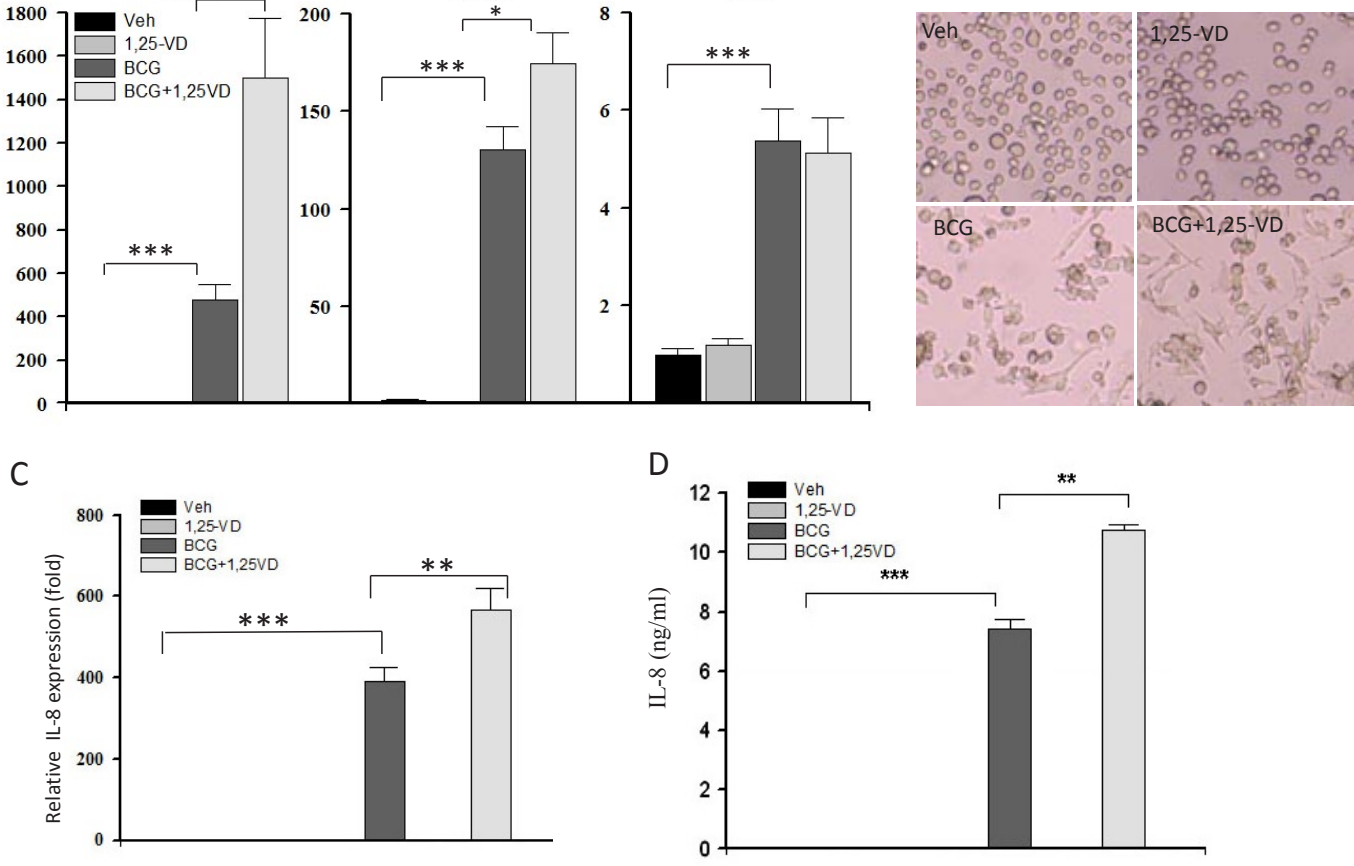

Figure 4: 1,25-VD promotes BCG induced THP-1 differentation/maturation. (A) THP-1 cells were treated with vehicle, 1.25 VD, BCG, or combination overnight. Cells were collected for total RNA extraction. The gene expression levels of macrophage markers, CD163, CHIT1, and CH68 were determined by real-time PCR assay. (B) Representative photos display a morphological alteration after treatments were shown. (C) The expression of IL- 8 after the treatment was detected by the real-time PCR and ELISA assays.

A

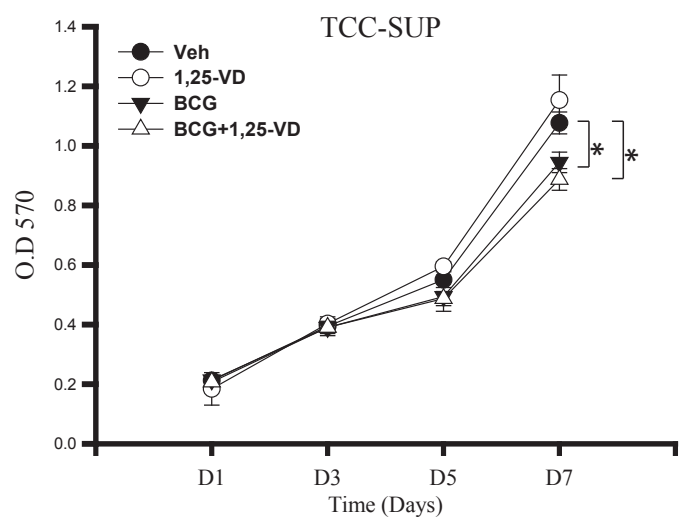

B

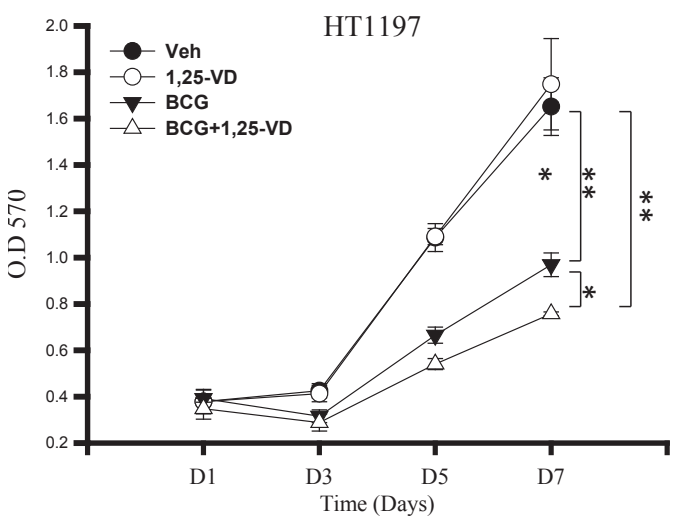

Figure 5: BCG-induced macrophage cytotoxcity against BCa cells. THP-1 cells were treated with vehicle control, 1,25-VD, BCG and in combination for 24 hours. The cell supernatants were collected and analyzed for their cytotoxicity against TCC-SUP BCa cells (A) and HT-1197 BCa cells (B). Cell viability was determined by 7-day MTT assay. For statistical analysis, differences in mean values between the two groups were analyzed by two-tailed Student's test. Results are the mean \pm SEM of three experiments. 
synergistically for higher therapeutic efficacy has been used in clinics. In this current study, we demonstrated that BCG and 1,25-VD combination therapy leads to a better treatment efficacy than mono-therapy, which is clinically significant. First, a significant number of $\mathrm{BCa}$ patients fail respond to $\mathrm{BCG}$, and $\mathrm{BCG}$ therapy commonly triggers local irritation effects and in rare cases, systemic toxicity. Second, the major limitation of systemic administration of pharmacologic doses of $1,25-\mathrm{VD}$ is hypercalcemia side effect. Thus, this intravesical instillation of BCG and 1,25-VD combination therapy offers a unique opportunity to improve current BCG immunotherapy while avoiding the side effects caused by a single regimen at high concentration. In our study, we found that 1,25-VD can promote BCG-induced immune response; thereby, possibly, we can lower BCG concentration to reduce BCGassociated side effects without losing its potency by coadministration of 1,25-VD. Importantly, the intravesical administration of 1,25 -VD provides the unique advantage that allows the use of high 1,25-VD concentration locally while avoiding hypercalcemia induced by high doses of 1,25 -VD given systemically. In this current study, we chose to combine 1,25-VD at high concentration $100 \mathrm{~nm}$ with $1 \times 10^{6} \mathrm{CFU}$ BCG, the lower end of BCG concentration used in most animal studies [30, 34-36]. We showed that the combination treatment indeed has better efficacy than BCG alone. We did not observe any obvious toxicity or weight loss in the combination treated mice. This pre-clinical study suggests that we can reduce BCG concentration without compromising its anti-tumor effects by combining it with a higher dosage of 1,25 -VD intravesically, through the mechanism involved in either enhancing innate immune responses [23] or antitumor effects [37]. A thorough pre-clinical study to determine the optimal combination doses is of great interest.

Even though we chose a lower concentration of

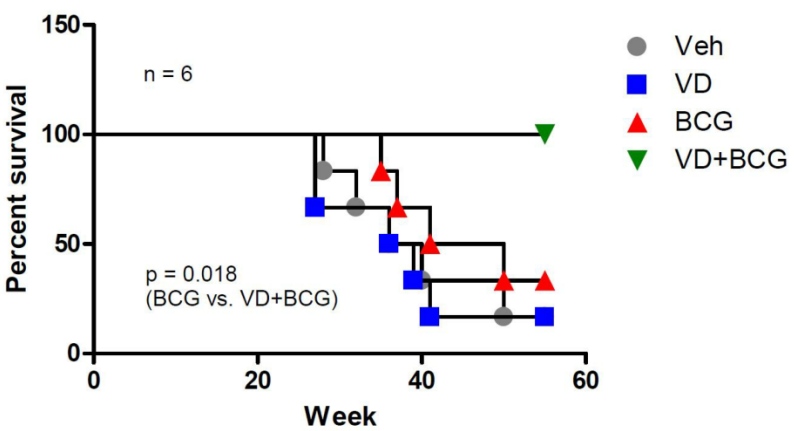

Figure 6: $\mathrm{BCG}+1,25-\mathrm{VD}$ increases the survival rate of BBN-induced BCa mice. 24 FVB female mice were initially given drinking water containing BBN at 6 weeks of age for 12 weeks' treatment. Subsequent to BBN treatment, 6 mice per group were subjected weekly to intravesical instillation of 100 $\mu \mathrm{l}$ PBS containing vehicle (ethanol), $100 \mathrm{nM} 1,25-\mathrm{VD}, 1 \times 10^{6}$ CFU BCG, or $100 \mathrm{nM} 1,25$-VD plus $1 \times 10^{6}$ CFU BCG. Mouse survival data were recorded weekly. The difference in survival rate was analyzed by Log-rank (Mantel-Cox) test.
BCG $\left(10^{6} \mathrm{CFU}\right)$ in our study, we expected to see BCG's anti-tumor effect. However, in this $\mathrm{BBN}-\mathrm{BCa}$ mouse model, BCG treatment can only slightly improve mice survival. In contrast to MB49 syngeneic model, one of the most common mouse models used for BCG therapy study, $10^{-6}$ to $10^{-7} \mathrm{CFU}$ BCG can improve MB-49 BCa mice survival [38]. One potential explanation for this reduced $\mathrm{BCG}$ efficacy might be associated with less availability of free fibronectin in this BBN-BCa model in which BCG was given at a very early stage of disease; fewer pre-cancer/cancer lesions are present, thereby less extracellular matrix fibronectins can be released. In contrast to the MB49 mouse model, the mice bladder walls are wounded first to allow MB49 BCa cells to adhere. This wounding process might also facilitate the fibronectin release. Interestingly, 1,25-VD was reported to increase fibronectin expression through a transcriptional regulation [39], which could then facilitate BCG internalization and yield better treatment efficacy.

IL-8 is a key prognostic marker for BCG immunotherapy for BCa [14, 20, 40-42]. A clinical study reported that patients who have detectable IL-8 in their urine after intravesical BCG treatment have a significantly higher disease-free survival [13]. Our data shows an increased IL-8 level induced by BCG and 1,25-VD combination therapy, thus enhancing the innate immune cell recruitment that promotes therapeutic efficacy. This result implies that a higher level of IL-8 in the bladder microenvironment is critical for BCG treatment. Therefore, to retain IL-8, for a longer period in the bladder potentially could be a strategy to improve BCG therapy. Previously, Zaharoff et al [35] demonstrated that intravesical instillation of chitosan and IL-12 exhibited a better therapeutic effect than BCG only, in which chitosan serves as an adhesive adjuvant and IL-12 as an immunotherapeutic agent with high potential to cure cancers [43]. Chitosan is cationic-charged and posses mucoadhesive properties, which enhance the adhesion of drug to negatively-charged bladder mucosa. In addition, its high viscosity can also enhance drug retention and promote drug permeability into urothelium by loosening the epithelial gap junctions [44]. Therefore, a combination of chitosan with Il-8 intravesical treatment could be a potential combination immunotherapy that needs further investigation.

The regulation of IL-8 production by vitamin D may vary under different physiological and pathological conditions. Our previous study suggested that $1,25-\mathrm{VD}$ can suppress IL-8 production of prostate cancer cells via blocking NF-kappaB activation [45]. However, in this current study we found that 1,25-VD promotes IL-8 production only in the presence of $\mathrm{BGC}$, suggesting this 1,25 -VD promoting IL- 8 is indirectly mediated through BCG-induced pathway. It is known that BCG binds to TLRs [46], which then induce the expression of an array of inflammatory cytokine genes, including IL-8 [47]. 
This activation of TLR signal by BCG could also activate vitamin D signal by enhancing the expression of VDR and CYP27beta1, and then inducing vitamin D downstream target expression such as anti-bacterial cathelicidins [48]. It is reported that cathelicidins, such as LL-37, can induce IL-8 production [49]. Therefore, the induction of IL- 8 by co-treating $1,25-\mathrm{VD}$ could be mediated indirectly via the activation of BCG-TLR downstream targets.

In summary, our study demonstrated a novel mechanism by which 1,25-VD promotes BCG-induced anti-BCa activity and this combination of BCG with 1,25VD provides a platform for improving BCG efficacy.

\section{MATERIALS AND METHODS}

\section{Cell lines and reagents}

Human urothelial immortalized cell line SV-HUC, low grade BCa cells HT1197 and high grade BCa cells TCC-SUP (Grade IV), T24 (Grade III) based on SRCCM alignment [26] and human monocyte cell line THP-1 were purchased from the American Type Culture Collection. Human neutrophil-like cell line HL-60 was a gift from Dr. Nazzarreno Ballatori at the University of Rochester. All the cell lines were cultured in RPMI-1640 medium with $10 \%$ heat-inactivated fetal bovine serum and L-glutamine (10 mM; Gibco). BCG was purchased from Sanofi Pasteur. 1,25-VD was purchased from Sigma Aldrich.

\section{Western blot assay}

Western blotting analysis was performed as previously described [27]. Briefly, cell lysate was prepared from each cell sample, and equal amounts of protein were resolved by electrophoresis in $10 \%$ or $15 \%$ SDSPAGE gels and transferred to polyvinylidene difluoride membrane (Millipore, Bedford, MA). Antibodies used are human VDR (N-20; Santa Cruz Biotechnology, Santa Cruz, CA), and beta-actin (Santa Cruz Biotechnology, Santa Cruz, CA).

\section{ELISA assay}

$2 \times 10^{5} \mathrm{BCa}$ cells or THP-1 cells were seeded in each well of a 24-well plate. The cells were treated with vehicle, $100 \mathrm{nM} 1,25-\mathrm{VD}, 2 \times 10^{6} \mathrm{CFU} / \mathrm{mL}$ BCG, or a combination of $100 \mathrm{nM} \mathrm{1,25-VD}$ and $2 \times 10^{6} \mathrm{CFU} /$ $\mathrm{mL}$ BCG. After 24 hours of treatment, the conditioned medium were harvested, filtered through a $0.22 \mu \mathrm{m}$ filter, and stored in $-80^{\circ} \mathrm{C}$ for determination of pro-inflammatory cytokine levels including IL-8, IL-6, and TNF-alpha by ELISA assay (eBioScience).

\section{Transwell migration assay}

Migration of macrophage was examined using a transwell assay. Thp-1 cells were cultured in serum free RPMI medium overnight, then $5 \times 10^{5}$ cells were added to each transwell insert with pore size $5 \mu \mathrm{m}$ (Corning Inc.). The lower chambers, serving as the chemo-attractant source, were loaded with $500 \mu$ conditioned media collected from the TCC-SUP cells treated with vehicle, $100 \mathrm{nM} 1,25-\mathrm{VD}, 5 \times 10^{5} \mathrm{CFU}$ BCG, or combination of BCG and 1,25-VD. Conditioned media were prepared by exposing the TCC-SUP cells to two hours of drug treatment, withdrawing the media and replacing them with fresh RPMI containing 10\% FBS and incubating for 22 hours. After 24 hours, the infiltrating cells in the lower chamber were collected and counted. $10 \mu \mathrm{g} / \mathrm{ml} \mathrm{IgG} \mathrm{or}$ anti-human IL-8 neutralizing antibody (R\&D systems) was added in the lower chamber to neutralize the IL-8 function in cell migration.

\section{Cytotoxicity assay}

HT1197 and TCC-SUP cells were seeded in 96-well culture plates. Cells were treated with diluted conditioned medium derived from supernatant of BCG and/or 1,25VD treated-THP-1 cells. Cell growth rate was determined by MTT assay. Briefly, culture media were replaced with fresh DMEM medium containing MTT stock solution and incubated at $37^{\circ} \mathrm{c}$ for two hours. Medium was then removed, dissolving solvent DMSO was added, and absorbance was measured at a wavelength of $570 \mathrm{~nm}$ with background subtraction at $660 \mathrm{~nm}$.

\section{RNA isolation and quantitative polymerase chain reaction (qPCR)}

Total RNA was isolated with Trizol (Invitrogen). Reverse transcription was performed with iScript Master Mix (BioRad). Quantitative RT-PCR was performed as previously described using $\mathrm{iQ}^{\mathrm{TM}} \mathrm{SYBR}{ }^{\circledR}$ Green Supermix with CFX96 real-time PCR detection system (Bio-Rad). Gene expression was analyzed by using CFX manager software normalizing the expression to two housekeeping genes, beta-actin and GAPDH. Primer design for CD68, CD163, CHIT-1, and IL-8 is from the PrimerBank (http:// pga.mgh.harvard.edu/primerbank).

\section{BBN-induced BCa mouse model and intravesical therapy of 1,25-VD and BCG}

All animal procedures were approved by the University Committee on Animal Resources of the University of Rochester and were in accordance with the National Institutes of Health guidelines. To generate a 
carcinogen-induced BCa model in mice, 24 FVB female mice at six weeks of age were given drinking water $\mathrm{ad}$ libitum containing $0.05 \%$ BBN (TCI America, Portland, OR) twice a week for 12 weeks. After completing BBN treatment, mice were subjected to four treatments of intravesical instillation: (1) vehicle control (ethanol); (2) $100 \mu \mathrm{M}$ 1,25-VD; (3) $10^{6}$ CFU BCG; or (4) $100 \mu \mathrm{M}$ 1,25VD combined with $10^{6} \mathrm{CFU}$ BCG. The drugs remained in the bladder lumens for one hour while animals were kept under isoflurane anesthesia. Intravesical treatments were administered once a week for six weeks. The mice survival rate was monitored.

\section{Statistical analysis}

Differences in mean values between the two (control) groups were analyzed by a two-tailed Student's $\mathrm{T}$ test. Differences in the survival rates of mice with $\mathrm{BCa}$ between the two groups were analyzed by a Log-rank (Mantel-Cox) test. P-values less than 0.05 were considered statistically significant.

\section{ACKNOWLEDGEMENTS}

This study was supported by grants funded by The Elsa U. Pardee Foundation and Roswell Cancer Institute/ James P Wilmot Cancer Center of the University of Rochester Medical Center.

\section{REFERENCES}

1. Jemal A, Siegel R, Xu J and Ward E. Cancer statistics, 2010. CA Cancer J Clin. 2010; 60(5):277-300.

2. Botteman MF, Pashos CL, Redaelli A, Laskin B and Hauser R. The health economics of bladder cancer: a comprehensive review of the published literature. Pharmacoeconomics. 2003; 21(18):1315-1330.

3. Sylvester RJ, van der Meijden AP, Oosterlinck W, Witjes JA, Bouffioux C, Denis L, Newling DW and Kurth K. Predicting recurrence and progression in individual patients with stage Ta T1 bladder cancer using EORTC risk tables: a combined analysis of 2596 patients from seven EORTC trials. Eur Urol. 2006; 49(3):466-465; discussion 475-467.

4. Morales A, Eidinger D and Bruce AW. Intracavitary Bacillus Calmette-Guerin in the treatment of superficial bladder tumors. The Journal of urology. 1976; 116(2):180183.

5. Kresowik TP and Griffith TS. Bacillus Calmette-Guerin immunotherapy for urothelial carcinoma of the bladder. Immunotherapy. 2009; 1(2):281-288.

6. Herr HW, Schwalb DM, Zhang ZF, Sogani PC, Fair WR, Whitmore WF, Jr. and Oettgen HF. Intravesical bacillus Calmette-Guerin therapy prevents tumor progression and death from superficial bladder cancer: ten-year follow- up of a prospective randomized trial. J Clin Oncol. 1995; 13(6):1404-1408.

7. Ratliff TL, Palmer JO, McGarr JA and Brown EJ. Intravesical Bacillus Calmette-Guerin therapy for murine bladder tumors: initiation of the response by fibronectinmediated attachment of Bacillus Calmette-Guerin. Cancer Res. 1987; 47(7):1762-1766.

8. Kuroda K, Brown EJ, Telle WB, Russell DG and Ratliff TL. Characterization of the internalization of bacillus CalmetteGuerin by human bladder tumor cells. J Clin Invest. 1993; 91(1):69-76.

9. de Boer EC, de Jong WH, van der Meijden AP, Steerenberg PA, Witjes F, Vegt PD, Debruyne FM and Ruitenberg EJ. Leukocytes in the urine after intravesical BCG treatment for superficial bladder cancer. A flow cytofluorometric analysis. Urol Res. 1991; 19(1):45-50.

10. Ludwig AT, Moore JM, Luo Y, Chen X, Saltsgaver NA, O'Donnell MA and Griffith TS. Tumor necrosis factorrelated apoptosis-inducing ligand: a novel mechanism for Bacillus Calmette-Guerin-induced antitumor activity. Cancer Res. 2004; 64(10):3386-3390.

11. Luo Y, Yamada H, Evanoff DP and Chen X. Role of Th1-stimulating cytokines in bacillus Calmette-Guerin (BCG)-induced macrophage cytotoxicity against mouse bladder cancer MBT-2 cells. Clinical and experimental immunology. 2006; 146(1):181-188.

12. Bettex-Galland M, Studer UE, Walz A, Dewald B and Baggiolini M. Neutrophil-activating peptide-1/interleukin-8 detection in human urine during acute bladder inflammation caused by transurethral resection of superficial cancer and bacillus Calmette-Guerin administration. European urology. 1991; 19(2):171-175.

13. Thalmann GN, Sermier A, Rentsch C, Mohrle K, Cecchini MG and Studer UE. Urinary Interleukin- 8 and 18 predict the response of superficial bladder cancer to intravesical therapy with bacillus Calmette-Guerin. The Journal of urology. 2000; 164(6):2129-2133.

14. Rabinowitz R, Smith DS, Tiemann DD and Hudson MA. Urinary interleukin-8/creatinine level as a predictor of response to intravesical bacillus Calmette-Guerin therapy in bladder tumor patients. The Journal of urology. 1997; 158(5):1728-1731; discussion 1731-1722.

15. Yamada $H$, Matsumoto $S$, Matsumoto $T$, Yamada $T$ and Yamashita U. Murine IL-2 secreting recombinant Bacillus Calmette-Guerin augments macrophage-mediated cytotoxicity against murine bladder cancer MBT-2. The Journal of urology. 2000; 164(2):526-531.

16. Brandau $\mathrm{S}$ and Suttmann $\mathrm{H}$. Thirty years of BCG immunotherapy for non-muscle invasive bladder cancer: a success story with room for improvement. Biomed Pharmacother. 2007; 61(6):299-305.

17. Messing EM. Words of wisdom. Re: Preexisting BCGspecific $\mathrm{T}$ cells improve intravesical immunotherapy for bladder cancer. Eur Urol. 2012; 62(5):935-936. 
18. Biot C, Rentsch CA, Gsponer JR, Birkhauser FD, Jusforgues-Saklani H, Lemaitre F, Auriau C, Bachmann A, Bousso P, Demangel C, Peduto L, Thalmann GN and Albert ML. Preexisting BCG-specific T cells improve intravesical immunotherapy for bladder cancer. Sci Transl Med. 2012; 4(137):137ra172.

19. Lamm DL, van der Meijden PM, Morales A, Brosman SA, Catalona WJ, Herr HW, Soloway MS, Steg A and Debruyne FM. Incidence and treatment of complications of bacillus Calmette-Guerin intravesical therapy in superficial bladder cancer. The Journal of urology. 1992; 147(3):596-600.

20. Thalmann GN, Dewald B, Baggiolini M and Studer UE. Interleukin- 8 expression in the urine after bacillus Calmette-Guerin therapy: a potential prognostic factor of tumor recurrence and progression. The Journal of urology. 1997; 158(4):1340-1344.

21. Rawls WH, Lamm DL, Lowe BA, Crawford ED, Sarosdy MF, Montie JE, Grossman HB and Scardino PT. Fatal sepsis following intravesical bacillus Calmette-Guerin administration for bladder cancer. The Journal of urology. 1990; 144(6):1328-1330.

22. Nnoaham KE and Clarke A. Low serum vitamin D levels and tuberculosis: a systematic review and meta-analysis. Int J Epidemiol. 2008; 37(1):113-119.

23. Liu PT, Stenger S, Li H, Wenzel L, Tan BH, Krutzik SR, Ochoa MT, Schauber J, Wu K, Meinken C, Kamen DL, Wagner M, Bals R, Steinmeyer A, Zugel U, Gallo RL, et al. Toll-like receptor triggering of a vitamin D-mediated human antimicrobial response. Science. 2006; 311(5768):17701773.

24. Sahin MO, Canda AE, Yorukoglu K, Mungan MU, Sade $\mathrm{M}$ and Kirkali Z. 1,25 Dihydroxyvitamin D(3) receptor expression in superficial transitional cell carcinoma of the bladder: a possible prognostic factor? Eur Urol. 2005; 47(1):52-57.

25. Mondul AM, Weinstein SJ, Mannisto S, Snyder K, Horst RL, Virtamo J and Albanes D. Serum vitamin D and risk of bladder cancer. Cancer Res. 2010; 70(22):9218-9223.

26. Dancik GM, Ru Y, Owens CR and Theodorescu D. A framework to select clinically relevant cancer cell lines for investigation by establishing their molecular similarity with primary human cancers. Cancer Res. 2011; 71(24):73987409.

27. Hsu JW, Yasmin-Karim S, King MR, Wojciechowski JC, Mickelsen D, Blair ML, Ting HJ, Ma WL and Lee YF. Suppression of prostate cancer cell rolling and adhesion to endothelium by 1alpha,25-dihydroxyvitamin D3. The American journal of pathology. 2011; 178(2):872-880.

28. de Reijke TM, Vos PC, de Boer EC, Bevers RF, de Muinck Keizer WH, Kurth KH and Schamhart DH. Cytokine production by the human bladder carcinoma cell line T24 in the presence of bacillus Calmette-Guerin (BCG). Urol Res. 1993; 21(5):349-352.

29. Esuvaranathan K, Alexandroff AB, McIntyre M, Jackson
AM, Prescott S, Chisholm GD and James K. Interleukin-6 production by bladder tumors is upregulated by BCG immunotherapy. The Journal of urology. 1995; 154(2 Pt 1):572-575.

30. Suttmann H, Riemensberger J, Bentien G, Schmaltz D, Stockle M, Jocham D, Bohle A and Brandau S. Neutrophil granulocytes are required for effective Bacillus CalmetteGuerin immunotherapy of bladder cancer and orchestrate local immune responses. Cancer Res. 2006; 66(16):82508257.

31. Fabriek BO, van Bruggen R, Deng DM, Ligtenberg AJ, Nazmi K, Schornagel K, Vloet RP, Dijkstra CD and van den Berg TK. The macrophage scavenger receptor CD163 functions as an innate immune sensor for bacteria. Blood. 2009; 113(4):887-892.

32. Luo Y and Knudson MJ. Mycobacterium bovis bacillus Calmette-Guerin-induced macrophage cytotoxicity against bladder cancer cells. Clin Dev Immunol. 2010; 2010:357591.

33. Hsu JW, Hsu I, Xu D, Miyamoto H, Liang L, Wu XR, Shyr $\mathrm{CR}$ and Chang C. Decreased tumorigenesis and mortality from bladder cancer in mice lacking urothelial androgen receptor. The American journal of pathology. 2013; 182(5):1811-1820.

34. Luo Y, Han R, Evanoff DP and Chen X. Interleukin-10 inhibits Mycobacterium bovis bacillus Calmette-Guerin (BCG)-induced macrophage cytotoxicity against bladder cancer cells. Clinical and experimental immunology. 2010; 160(3):359-368.

35. Zaharoff DA, Hoffman BS, Hooper HB, Benjamin CJ, Jr., Khurana KK, Hance KW, Rogers CJ, Pinto PA, Schlom J and Greiner JW. Intravesical immunotherapy of superficial bladder cancer with chitosan/interleukin-12. Cancer Res. 2009; 69(15):6192-6199.

36. Reece ST, Nasser-Eddine A, Dietrich J, Stein M, Zedler U, Schommer-Leitner S, Ottenhoff TH, Andersen P and Kaufmann SH. Improved long-term protection against Mycobacterium tuberculosis Beijing/W in mice after intradermal inoculation of recombinant BCG expressing latency associated antigens. Vaccine. 2011; 29(47):8740-8744.

37. Konety BR, Lavelle JP, Pirtskalaishvili G, Dhir R, Meyers SA, Nguyen TS, Hershberger P, Shurin MR, Johnson CS, Trump DL, Zeidel ML and Getzenberg RH. Effects of vitamin D (calcitriol) on transitional cell carcinoma of the bladder in vitro and in vivo. J Urol. 2001; 165(1):253-258.

38. Ratliff TL. Role of animal models in understanding intravesical therapy with bacille Calmette-Guerin. Clin Infect Dis. 2000; 31 Suppl 3:S106-108.

39. Polly P, Carlberg C, Eisman JA and Morrison NA. Identification of a vitamin D3 response element in the fibronectin gene that is bound by a vitamin D3 receptor homodimer. J Cell Biochem. 1996; 60(3):322-333.

40. de Boer EC, Somogyi L, de Ruiter GJ, de Reijke TM, Kurth $\mathrm{KH}$ and Schamhart DH. Role of interleukin- 8 in onset of 
the immune response in intravesical BCG therapy for superficial bladder cancer. Urol Res. 1997; 25(1):31-34.

41. Kumar A, Dubey D, Bansal P, Mandhani A and Naik S. Urinary interleukin-8 predicts the response of standard and low dose intravesical bacillus Calmette-Guerin (modified Danish 1331 strain) for superficial bladder cancer. The Journal of urology. 2002; 168(5):2232-2235.

42. Sagnak L, Ersoy H, Ozok U, Senturk B, Ercil H, Bahar $\mathrm{G}$ and Ozturk E. Predictive value of urinary interleukin- 8 cutoff point for recurrences after transurethral resection plus induction bacillus Calmette-Guerin treatment in nonmuscle-invasive bladder tumors. Clin Genitourin Cancer. 2009; 7(2):E16-23.

43. Cheever MA. Twelve immunotherapy drugs that could cure cancers. Immunol Rev. 2008; 222:357-368.

44. Dodane V, Amin Khan M and Merwin JR. Effect of chitosan on epithelial permeability and structure. Int J Pharm. 1999; 182(1):21-32.

45. Bao BY, Yao J and Lee YF. 1alpha, 25-dihydroxyvitamin D3 suppresses interleukin-8-mediated prostate cancer cell angiogenesis. Carcinogenesis. 2006; 27(9):1883-1893.

46. Larue H, Ayari C, Bergeron A and Fradet Y. Tolllike receptors in urothelial cells-targets for cancer immunotherapy. Nature reviews Urology. 2013; 10(9):537545.

47. Kawai T and Akira S. Signaling to NF-kappaB by Toll-like receptors. Trends in molecular medicine. 2007; 13(11):460469.

48. Khoo AL, Chai LY, Koenen HJ, Oosting M, Steinmeyer A, Zuegel U, Joosten I, Netea MG and van der Ven AJ. Vitamin $\mathrm{D}(3)$ down-regulates proinflammatory cytokine response to Mycobacterium tuberculosis through pattern recognition receptors while inducing protective cathelicidin production. Cytokine. 2011; 55(2):294-300.

49. Choi KY and Mookherjee N. Multiple immune-modulatory functions of cathelicidin host defense peptides. Frontiers in immunology. 2012; 3:149. 